\title{
Four-Dimensional of Japan Earthquake-Tsunami Disaster from Rapid Eye Satellite Data
}

\author{
Maged Marghany \\ Faculty Geospatial and Real Estate, Geomatika University College, Lot 5-5-7, 5th Floor, Prima Peninsular, \\ Jalan Setiawangsa 11, Taman Setiawangsa, 54200, Kuala Lumpur, WP Kuala Lumpur, Malaysia \\ *Corresponding Author: Maged Marghany. Faculty Geospatial and Real Estate, Geomatika University \\ College, Lot 5-5-7, 5th Floor, Prima Peninsular, Jalan Setiawangsa 11, Taman Setiawangsa, 54200, \\ Kuala Lumpur, WP Kuala Lumpur, Malaysia
}

\begin{abstract}
This study is a novel work proposed to model a four-dimensional form. In fact, all the computer models are constrained to only three-dimensional shapes. In this study, hologram interferometric with 4-D phase unwrapping is used to reconstruct fourth-dimension of Japan's tsunami impacts. The data are used that involved two Rapid Eye images. The hybrid genetic algorithm is used for 4-D phase unwrapping. The results show that the hologram Interferometric an excellent tool for reconstructing tsunami chaotic effects of land uses from Rapid Eye data. The results show that the greatest damage has occurred in the urban, road network, and infrastructures discriminated. In conclusion, the optical hologram Interferometric is an appropriate algorithm for chaotic 4-D automatic detection of tsunami impacts in Rapid Eye data.
\end{abstract}

Keywords: Fourth-dimensional, Hologram interferometric, RapidEye satellite data, Hybrid Genetic.

\section{INTRODUCTION}

The four-dimensional reconstruction is still in early stage of development for remote sensing technology and its application to natural disasters such as a tsunami. In spite of there are many attempts efforts are set up to reconstruct the universe in multidimensional [1-4] [33]. Marghany [33] has established a novel concept to model the tsunami wave propagation in 4-D. This work is an extent of Marghany [33].

Tsunamis are water waves in which the reinstating energy is gravity and the wavelength is superior in the ocean depth. Dissimilar shorter wavelength instabilities, the water particle motion spreads all the way through the water column. Continuously future the catastrophe of the Indian Ocean tsunami of Boxing Day 2004 and Japanese 2011, research in tsunami geoscience has improved obviously [23]. Scientists have endeavored to realize the mechanisms of the large scale of both the Indian Ocean tsunami of 2004 and the Japanese 2011 tsunami [23][24]. Nevertheless, according to Marghany [ 26], the great efforts have been made by scientists since Boxing day 2004 do not prevent the occurrence of the Japanese tsunami with great disaster occurred. Nevertheless, on March 11, 2011, a magnitude of $\mathrm{M}_{\mathrm{w}} 9.0$ earthquake struck off the coast of Honshu, Japan, sparking a tsunami that not only devastated the island nation (Figure 1), but also caused destruction and fatalities in other parts of the world, including Pacific islands i.e. Hawaii, the Philippines, and Mexico and the United State (U.S.) West Coast [4] [Marghany [26]. With this regard, in 140 years, the earthquake was the strongest which run into 81 miles (130 kilometers) off the coast of Sendai at 2:46 p.m. local time.

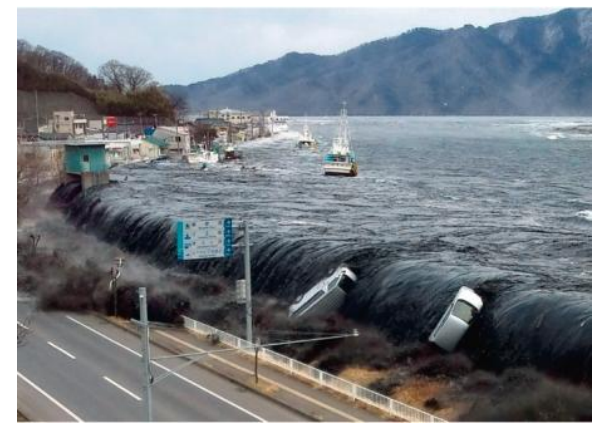

Figure1. Disaster due to 2011 Japan Earthquake-Tsunami 


\subsection{Generation of 2011 Japan 's Tsunami}

The massive destructive 2011 Japan 's tsunami is generated due to the largest fault slip occurrence. With this regard, the fault or the boundary between two tectonic plates of the Pacific plate and a portion of the North American plate in the Japan trench slipped approximately about 164 feet (50 meters) (Figure 2). Other similarly large magnitude earthquakes, including the 9.1 Sumatra event in 2004, resulted in a 66-to-82 foot (20-to-25 meter) slip on the fault.

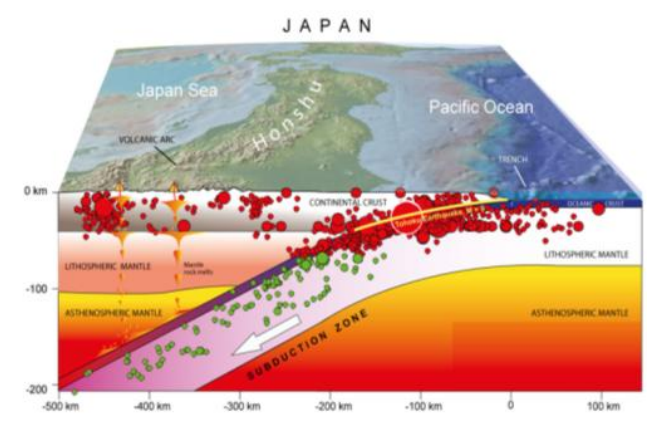

Figure2. Subduction of 2011 Japan earthquak.

A thick layer of clay is seated on the top of the Pacific plate, which is stepping pulled under a portion of the North American plate. By way of the Pacific, plate plunges into a trench off the coast of Japan, small portions of the clay grow smeared along the plate tectonics. That clay holds water, reducing it reasonably greasy, which was accountable for permitting the incredibly huge slip are declared near the trench (Figure 3).

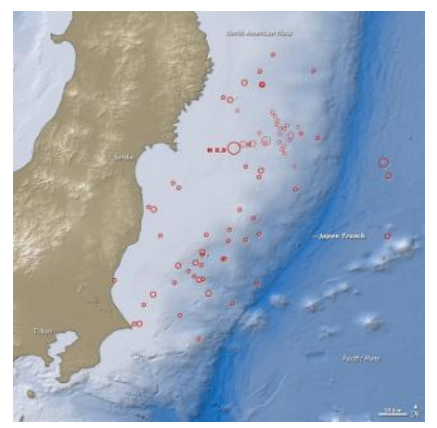

Figure3. Japan trench location

The abrupt slips throughout an earthquake can create massive quantities of heat and to expel sections of the fault zone itself. The earthquake formed a $600^{\circ}$ to $1,200^{\circ} \mathrm{C}$ temperature, which expanded the amount of friction. Then, fault slip was generated by clay lubrication. During 2011 Japan's earthquake, the plates are crushed together at this trench, which horizontal shift plunges up enough seawater to generate the killer tsunami that hit Japan.

\subsection{Path of 2011 Japan's Tsunami Propagation}

The tsunami waves propagated crosswise the Pacific, arriving in Alaska, Hawaii, and Chile. In Chile, some 11,000 miles $(17,000 \mathrm{~km})$ distant, the tsunami was 6.6 feet $(2$ meters $)$ high when they approached the shore (Figure 4). The surge of water brought an estimated five million tons of debris out to sea. Japanese docks and ships, and countless household items have arrived on U.S. and Canadian shores in the ensuing years. It is clear that the tsunami wave approached the South China Sea within 3 hours and moved into coastal waters of Malaysia within 6 hours (Figure 4).

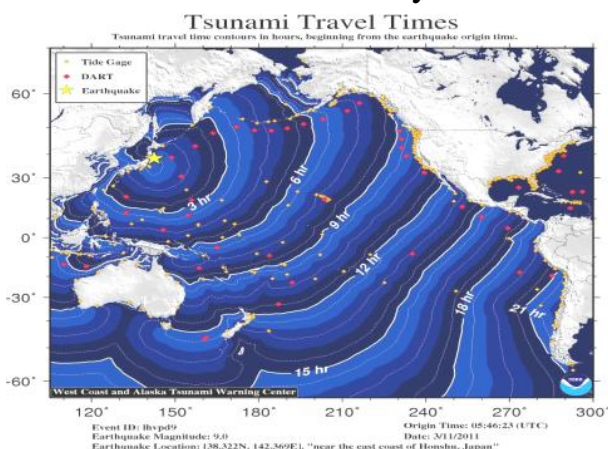

Figure4. Tsunami Travelling time and propagatio. 
In addition, Figure 5 shows the whole house drifted by the 2011 tsunami and current pattern of Pacific Ocean which traveled toward U.S coastal waters within 3 years (Figure 6).

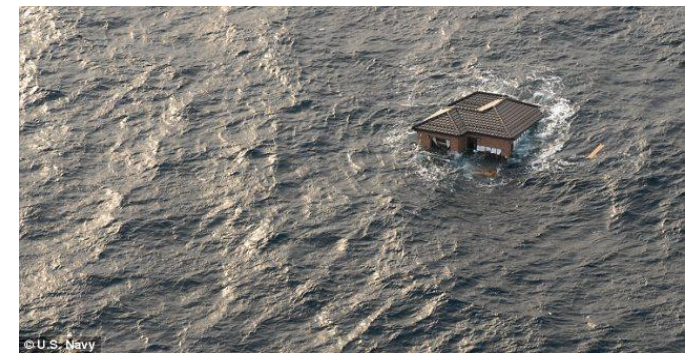

Figure5. Debris of house in the Pacific Ocean [28]

Figure 6 shows several thousand bodies were washed out to sea following the disaster and while most of the limbs will come apart and break down in the water, feet encased in shoes will float towards the coastal water of North America [28].

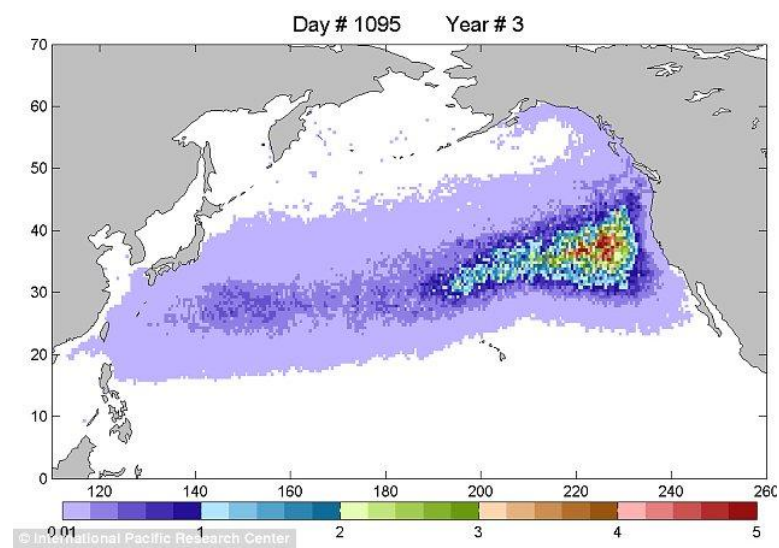

Figure6. Trajectory movements of 2011 Japan' tsunami debris [28]

\subsection{Why did a Whirlpool form in a Japanese Harbor after the Tsunami Hit?}

The main feature was observed during 2011 Japan's tsunami was a whirlpool (Figure 7). Whirlpools occur owing to the interface between running water, shallow sea bed and coastline 's geological features. In other words, there is great friction produced due to the interaction of water with shallow bathymetry. With this regard, the friction has reduced the speed of running water and generated

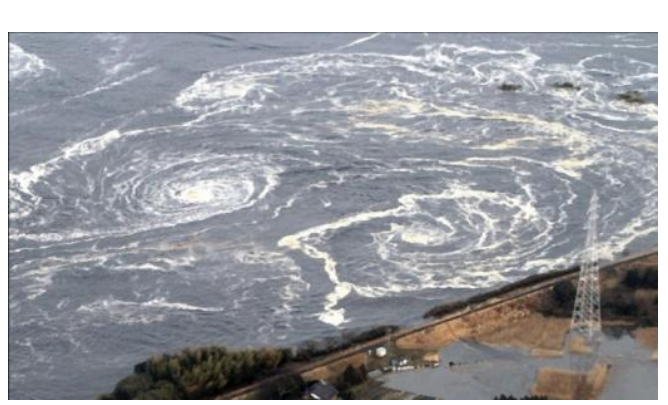
rotating turbulent flow in the shape of Whirlpools.

Figure7. Tsunami wave generated whirlpools along the coastal water of Japa.

\subsection{Energy Delivered from 2011 Japan's Tsunami}

Kitanidis [28] stated the surface energy of the seismic waves approximately of $1.9 \times 10^{17}$ joules have been delivered 2011 Japan's earthquake. This amount was double of the 2004 Indian Ocean tsunami. However, the seismic moment $\left(\mathrm{M}_{0}\right)$, of a physical size for 2011 Japan ' $\mathrm{s}$ tsunami of $3.9 \times 10^{22}$ joules, was slightly less than the 2004 Indian Ocean quake [30]. In addition, a peak ground acceleration was about $2.99 \mathrm{~g}\left(29.33 \mathrm{~m} / \mathrm{s}^{2}\right)$. The largest individual recording in Japan was $2.7 \mathrm{~g}$, in Miyagi Prefecture, $75 \mathrm{~km}$ from the epicenter; the highest reading in the Tokyo metropolitan area was $0.16 \mathrm{~g} \mathrm{[29-31].}$

\subsection{Four-Dimensional Theories and Concept}

Hitherto developmentally remote sensing technology disables to implement $\mathrm{n}$-dimensional and is just constrained to simulate three-dimensional of any object on the ground.Though, n-dimensional 
newly is an interesting theme for mathematicians and physicians. String theory, M-theory, and Supergravity are most well known n-dimensional theories. String theory, consequently, proposed that the universe is shaped in multiple dimensions: (i) height, (ii) width, and (iii) length compose three-dimensional space; and (iv) time contributes an entirety of four observable dimensions. String theories, nonetheless, continued the probability of ten dimensions - the remaining six of which human capability cannot depiction precisely. Thus supergravity theory that fuses the theories of supersymmetry and general relativity. Further, Supergravity theory has played an important part in verifying the basic of the $11^{\text {th }}$ dimension. To close, M-theory conjoins the five various string theories (along with a subsequently discarded challenge to fuse General Relativity and Quantum Mechanics called 11-D-Supergravity) into one theory [1][14].

M-theory, yonder hypothetically, can assume an assembly of developing a unified theory of all of the elementary potencies of nature. Challenges to contact M-theory for investigating typically converge on compactifying (i.e., theory with respect to one of its space-time dimensions) and its extra dimensions to construct candidate models of our four-dimensional world.Though, the geometry of 4-dimensional (4-D) space is abundant extra convoluted than that of 3-dimensional space, because of the extraordinary degree of freedom. Consequently,4-D involves 4-polytopes which are formulated of polyhedral (Figure 8). In addition, 4-D also contains 6 convex regular 4-polytopes, which are the analogs of the Platonic solids. Therefore, relaxing the conditions for reliability spawns an additional 58 convex uniform 4-polytopes, corresponding to the 13 semi-regular Archimedean solids in three dimensions [1,15]. Therefore, as 3-D beings, it cannot transfer freely in time, but in 4$\mathrm{D}$ it could be achievable. In this context, 4-D can distinguish three-dimensional and it cannot be conveyed to Euclidean space which suggests that fourth- dimension is spatial.Therefore, 4-D can be generated algebraically, by utilizing the regulations of vectors and coordinate geometry to a space in 4-D $[2,14,15]$.

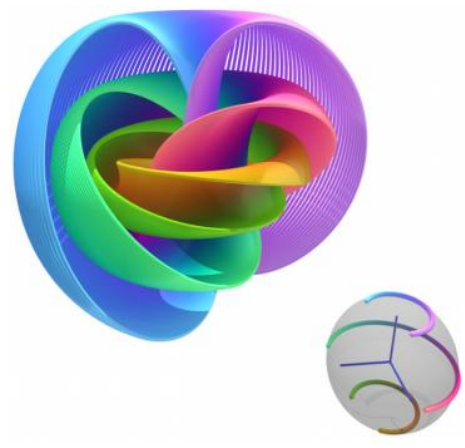

Figure8. The concept of Four-dimensional

A vector for instance, with four elements (a 4-tuple), particularly can be instigated to attitude as an object in 4-D. Space is a Euclidean space, which has a metric and norm. Further, all directions are handled as the same: the added dimension is incoherent from the other three. Consequently, 4-D space has an extra coordinate axis, orthogonal to the other three, which is usually labeled $w$ as compared to 3 -D. A position along the $w$ axis can be called spissitude. To comprehend the nature of 4-D. With this regard, space, a device called dimensional analogy is commonly employed. The dimensional analogy is the study of how $(n-1)$ dimensions correlate to $n$-dimensions and then supposing how $n$ dimensions would draw a parallel to $(n+1)$ dimensions $[2,15]$.

On the other hand, the n-dimensional establishment in the real world is still in a preliminary step. This is because the main limitation of human eyes to visualize objects in 2-D and by the assistance of computer graphic algorithms can step in 3-D. Though there are great advances in remote sensing technology, which can provide 3-D maps through LIDAR, TanDAM TerraSAR $X$ etc., the human eyes are restricted to view 2-D or 3-D objects and not able to be viewed in ndimension. In free space universe, there are parallel coordinates which can view the universe in $n$ dimensional. One of the accurate remote sensing technique for 3-D visualization is interferometry synthetic aperture radar techniques. Yet, the performance of the interferometric phase estimation suffers seriously from poor image coregistration. Interferogram filtering algorithms such as an adaptive contoured window, pivoting mean filtering, pivoting median filtering, and adaptive phase noise filtering are the main methods for the conventional InSAR interferometric phase estimation [16]. Recently, Marghany [12] implemented hologram interferometry for shoreline change. Marghany [13] introduced a new technique to reconstruct 4-D from hologram interferometry for International Journal of Research in Environmental Science (IJRES) 
optical remote sensing and ENVISAT ASAR data. However, these studies do not explain clearly the mathematical formula used to reconstruct 4-D. In fact, 4-D is required to formulate mathematical protocols to be implemented in several applications [15].

\subsection{Hypotheses and Objective}

The concern with the above perspective, we address the question is how to reconstruct 4-D from 3$\mathrm{D}$ ? This study postulates that 4-D can be implied from 3-D phase unwrapping of optical hologram interferometry. As a matter of fact, optical interferometry is a powerful approach to measure shifts of the stability of electromagnetic wavelength spectra. One significant limitation of common interferometric methods is that they require specular reflectors. This limitation can be removed by utilizing holography, allowing very small motions of arbitrary, diffusely reflecting, objects to be detected.

The main novelty of this study is to derive a new formula for 4-D hologram interferometry phase unwrapping Hybrid Genetic Algorithm (HGA). The main objective is to reconstruct fourthdimension of Japan's Tsunami impacts high-resolution Rapid Eye satellite data by optimization of 4D hologram interferometry.

Three hypotheses are examined: (i) 4-D can be retrieved from 3-D as 4-D is encoding of 3-D; (ii) hologram interferometry can reconstruct 4-D tsunami 's object detection in high-resolution Rapid Eye satellite data; and (iii) Hybrid Genetic Algorithm (HGA) can be used to optimize 4-D object reconstruction in Rapid Eye satellite data

\section{HOLOGRAM INTERFEROMETRY TO RECONSTRUCT FOURTH-DIMENSIONAL}

Consistent with Jones and Wykes [31] Holographic interferometry (HI) is a method which permits static and dynamic dislocations of objects with optically rough surfaces to be computed to optical interferometric accuracy (i.e., too a small percentage of a wavelength of electromagnetic spectra of visible bands). These quantities can be implemented to stress, strain and vibration analysis, besides to non-destructive testing[31-33].

For instance, fluid flow visualization can be implemented using the vibration of the electromagnetic wavelength through the fluid. In this regard, the fluid velocity changes can be represented by contours that indicate the deformation of the fluid surface.

Joud et al., [32] reported that holography is the two-phase procedures of digital recording of a diffracted electromagnetic wave which scattered from an object, and functioning of image rendering. This procedure can be accomplished with conventional photographic plates or with a digital sensor array, in digital holography.

The two fields will be indistinguishable when the verified field is covered by the electromagnetic waves scattered from the object. Uncertainty, nonetheless, a minor distortion has affected the object, the relative stages of the two light fields will modify, and it is possible to detect interference. This practice is recognized as holographic interferometry. The deformation, which is recorded from the holographic interferometry is represented as fringe patterns which are termed as 'frozen fringe' holography [31][32].

Following Marghany (2016), assume that $I_{1}$ and $I_{2}$ are the different acquisition times of two optical satellite data, for instance, RapidEye. Consequently, $I_{1} \in E_{1}$ and $I_{2} \in E_{2}$ where $E_{1} \notin E_{2}$ or $E_{1} \neq E_{2}$ as $E$ is electromagnetic spectra which presents in two RapidEye satellite data. Both electromagnetic spectra waves interfere at the surface of point in space or time. Hence, their amplitudes will add as a vector. If one of these is a plane wave pointing in the z-direction and the other is a spherical wave, then it can be described by

$$
\begin{aligned}
& I(x, y)=\left|E(x, y) \mathrm{e}^{\left(\mathrm{i} \phi \phi_{O}(x, y)\right)}+r(x, y) \mathrm{e}^{\left(\mathrm{i} \phi_{R}(x, y)\right)}\right| \\
& =\left(E_{2}(x, y) \mathrm{e}^{\left(\mathrm{i} \phi_{R}(x, y)\right)}\right)\left(r(x, y) \mathrm{e}^{\left(\mathrm{i} \phi_{R}(x, y)\right)}\right)^{*}+\left(E_{l}(x, y) \mathrm{e}^{(\mathrm{i} \phi O(x, y))}\right)\left(E(x, y) \mathrm{e}^{(\mathrm{i} \phi O(x, y))}\right)^{*} \\
& \left.\quad+\left(E_{1}(x, y) \mathrm{e}^{\left(\mathrm{i} \phi_{O}(x, y)\right)}\right)\right)\left(E_{2}(x, y) \mathrm{e}^{\left(\mathrm{i} \phi_{R}(x, y)\right)}\right)^{*}+\left(E_{2}(x, y) \mathrm{e}^{\left(\mathrm{i} \phi_{R}(x, y)\right)}\right)\left(E_{l}(x, y) \mathrm{e}^{\left(\mathrm{i} \phi_{O}(x, y)\right)}\right)^{*}
\end{aligned}
$$

where $\boldsymbol{E}$ is the complex amplitude of the object wave with real amplitude $E_{1}$ and phase $\phi O, \mathbf{E}_{2}$ is the complex amplitude of the reference wave with real amplitude $E_{2}$ and phase $\phi R$ and $*$ denotes the conjugate complex.

The phase changes due to deformation can be obtained by using 2-DFFT into the above equation [21] 


$$
F F T\{I\}=A\left(f_{x}+f_{y}\right)+B\left(f-f_{x}, f-f_{y}\right) B^{*}\left(f-f_{x}, f-f_{y}\right)
$$

where FFT denote the Fourier spectra and $f$ is the spatial frequency in the $x$ and $y$ directions, are the carry frequencies in the $x$ and $y$ directions, respectively [15]. Following De la Torre et al., [3], 4-D holographic interferometry can be given by

$$
\begin{aligned}
& \boldsymbol{F F T}\left\{\boldsymbol{I}_{N}\right\}=\sum_{N=1}^{4}\left[\boldsymbol{A}_{N}\left(f_{x}+f_{y}+f_{z}\right)+\boldsymbol{B}_{N}\left(f-f_{N x}, f-f_{N y}, f-f_{N z}\right)+\right. \\
& \left.\boldsymbol{B}_{N}{ }^{*}\left(f-f_{N x}, f-f_{N y}, f-f_{N z}\right)\right]
\end{aligned}
$$

where $N$ represents fourth -dimensional, $A_{N}$ is incoherent in 4-D holographic interferometry. $B_{N}$ and $B_{N}{ }^{*}$ are lobes for each illumination wavelength in RapidEye satellite data. The relative optical phase difference can be associated with a physical displacement through the sensitivity vector found in the hologram interferometry in two satellite data which can be expressed in 4-D as,

$$
\left(\begin{array}{c}
\Delta \Phi_{1} \\
\Delta \Phi_{2} \\
\Delta \Phi_{3} \\
\Delta \Phi_{4}
\end{array}\right)=\frac{2 \pi}{\lambda}\left(\begin{array}{llll}
\vec{d}_{1 i} & \vec{d}_{1 j} & \vec{d}_{1 k} & \vec{d}_{1 p} \\
\vec{d}_{2 i} & \vec{d}_{2 j} & \vec{d}_{2 k} & \vec{d}_{2 p} \\
\vec{d}_{3 i} & \vec{d}_{3 j} & \vec{d}_{3 k} & \vec{d}_{3 p} \\
\vec{d}_{4 i} & \vec{d}_{4 j} & \vec{d}_{4 k} & \vec{d}_{4 p}
\end{array}\right)\left(\begin{array}{l}
U \\
V \\
W \\
O
\end{array}\right)
$$

Following Marghany [15] where $d$ is the displacement in along orthogonal components of $U, V, W, O$, in $i, j, k$, and $p$, respectively. According to Marghany [15] Phase unwrapping can be extended into fourthdimensional by the given equation,

$$
\begin{aligned}
& \sum_{i, j, k, p} W_{i, j, k, p}^{x}\left|\Delta \phi_{i, j, k, p}^{x}-\Delta \psi_{i, j, k, p}^{x}\right|^{L}+\sum_{i, j, k, p} W_{i, j, k, p}^{y}\left|\Delta \phi_{i, j, k, p}^{y}-\Delta \boldsymbol{\psi}_{i, j, k, p}^{y}\right|^{L} \\
& +\sum_{i, j, k, p} W_{i, j, k, p}^{z}\left|\Delta \phi_{i, j, k, p}^{z}-\Delta \boldsymbol{\psi}_{i, j, k, p}^{z}\right|^{L}+\sum_{i, j, k, p} \boldsymbol{W}_{i, j, k, p}^{z}\left|\Delta \phi_{i, j, k, p}^{w}-\Delta \boldsymbol{\psi}_{i, j, k, p}^{w}\right|^{L}
\end{aligned}
$$

where $\Delta \phi$ and $\Delta \psi$ are the unwrapped and wrapped phase differences in $x, y, z, w$ respectively, and $W$ represents user-defined weights. The summations are carried out in both $x, y, z$, and $w$ directions over all $i, j, k$, and $p$ respectively [15].

\section{Hybrid Genetic Algorithm (HGA)}

The HGA algorithm relies on estimating the parameters of an $\mathrm{n}^{\text {th }}$ order-polynomial to approximate the unwrapped surface solution from the wrapped phase data (Figure 9) [7][15]. Any optimization problem using a genetic algorithm (GA) requires the problem to be coded into the GA syntax form, which is the chromosome form. In this problem, the chromosome consists of a number of genes where every gene corresponds to a coefficient in the $\mathrm{n}^{\text {th }}$-order surface fitting polynomial this can be extend into 4-D as follows [15],

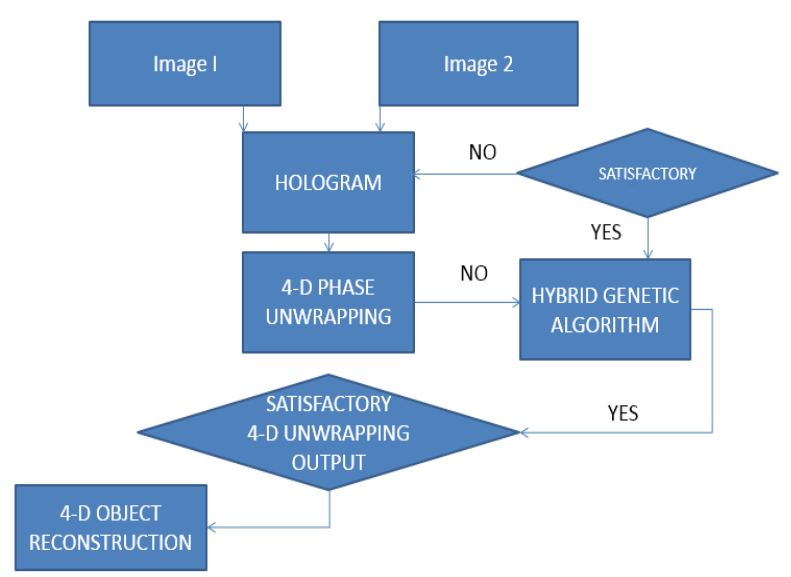

Figure9: 4-D procedures of holographic interferometry

$$
f:=n \rightarrow \sum_{p=0}^{n} \sum_{k=0}^{n} \sum_{j=0}^{n} \sum_{i=0}^{n} a_{i, j, k, p} \Delta \phi_{i, j, k, p}^{x} \Delta \phi_{i, j, k, p}^{y} \Delta \phi_{i, j, k, p}^{z} \Delta \phi_{i, j, k, p}^{w}
$$

where $a[0 \ldots . n]$ are the parameter coefficients which are retrieved by the genetic algorithm to approximated the unwrapped phase. Further, $i, j, k$, and $p$ are indices of the pixel location in the unwrapped phase in 4-D, respectively, $n$ is the number of coefficients. 


\subsection{Initial Solution and Population}

Following Karout [7], the initial solution is approximated using a 'polynomial Surface-fitting weighted least-square multiple regression' method. The initial population is then generated based on the initial solution. In doing so, every $a_{g}$ in each chromosome in the population, a small number relying on the accuracy of the gene that is added or subtracted to the value of the gene as given by $[14,15]$,

$a_{g}=a_{g}+( \pm 1)\left\{10^{\left[\log \left(a_{g}\right)+\Re\right]}\right\}$

where $a_{g}$ is the coefficient para meter stored in gene $g$, and $\Re$ is a random number generated between the values in 4-D.

\subsection{Record Pareto Optimal Solutions}

Calculate the objective values of chromosomes in the population and record the Pareto optimal solutions.

Definition: Pareto Optimal Solutions

Let $\phi_{i}, \phi_{i, j}, \phi_{i, j, k}, \phi_{i, j, k, p} \in F$, and $F$ is a feasible region in the 4-D coordinate. And $\phi_{0}$ is called the Pareto optimal solution to the minimization problem of 4-D phase unwrapping if the following conditions are satisfied.

1. If $f\left(\phi_{1}\right)$ is said to be partially greater than $f\left(\phi_{2}\right)$, i.e $f_{i}\left(\phi_{1}\right) \geq f_{i}\left(\phi_{2}\right), \forall N=1,2, \ldots, n$ and $f_{i}\left(\phi_{1}\right)>f_{i}\left(\phi_{2}\right), \exists N=1,2, \ldots, n$,

Then $\phi_{1}$ is said to be dominated by $\phi_{2}$.

2. If there is no $\phi \in F$ s.t. $\phi$ dominates $\phi_{0}$, then $\phi_{0}$ is the Pareto optimal solutions.

\subsection{Fitness Evaluation}

In this step, the quality of the solution is evaluated at every generation to determine the global optimum solution to the parameter estimation phase unwrapping problem $[17,19]$. Therefore, the genes on a chosen chromosome to evaluate the approximated phase value at coordinates $(i, j, k, p)$. Then, the obtained phase is subtracted from the contiguous pixel approximated phase value to retrieve the approximated unwrapped phase solution gradient. It is then subtracted from the gradient of the wrapped phase in the $i, j, k$ and $p$ direction[15,18].

\subsection{Crossover and Mutation}

Following Haupt and Haupt [6], the two-point greedy continuous crossover is implemented in the crossover operator. Therefore, the crossover is less problematic than the mutation operator [17]. Thus, mutation operator concerns deliberate changes to a gene at random, to keep variation in genes and to increase the probability of not falling into a local minimum solution. It involves exploring the search space for new better solutions [6]. This proposed operator uses a greedy technique which ensures only the best fit chromosome is allowed to propagate to the next generation $[5,7,15]$

\subsection{Phase Matching}

The accurate 4-D phase unwrapping can be obtained by phase matching algorithm which is suggested by Schwarz [20]. Consistent with Schwarz [20], the phase matching algorithm is matched the phase of wrapped phase with the unwrapped phase by the given equation

$$
\psi_{i, j, k, p}=\Delta \phi_{i, j, k, p}+2 \pi \rho\left[\frac{1}{2 \pi}\left(\hat{\Delta \phi}_{i, j, k, p}-\Delta \phi_{i, j, k, p}\right)\right]
$$

where $\psi_{i, j, k, p}$ is the phase matched unwrapped phase, $i, j$, and $k$ are the pixel positions in the quality phase map, $\Delta \phi_{i, j, k, p}$ is the given wrapped phase, $\Delta \hat{\phi}_{i, j, k, p}$ is the approximated unwrapped phase, $\rho[$. 
is a rounding function which is defined by $\rho[t]=\lfloor t+1 / 2\rfloor$ for $t \geq 0$ and $\rho[t]=\lfloor t-1 / 2\rfloor$ for $t<0$ and are $i, j, k$ and $p$ the pixel positions in $x$ and $y, z, w$ directions, respectively $[11,15,16,20]$.

\section{RESULTS AND DISCUSSION}

The massive tidal wave has created by 9.0 Richter magnitude earthquake and hit Japan with incredible force On March 11, 2011, causing catastrophic devastation. During and post the events, the Earth observation technology including buoys, satellites and other imaging devices have allowed scientists and the media to visualize and track the impact of the event, mitigating the loss of life and property and supporting effective rescue efforts. Figure 10 shows the two RapidEye satellite data were acquired over Japan on September 5, 2010, and March 12, 2011, respectively.

(a)

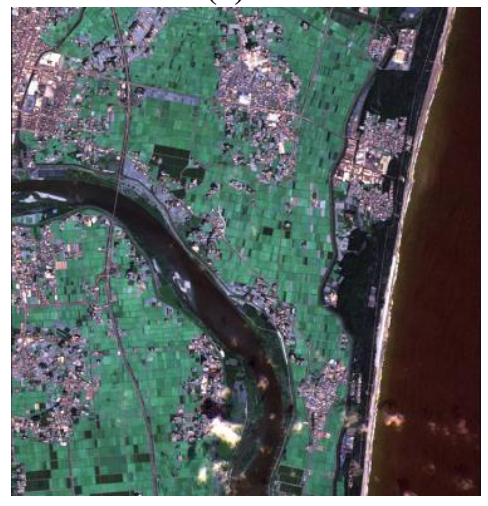

(b)

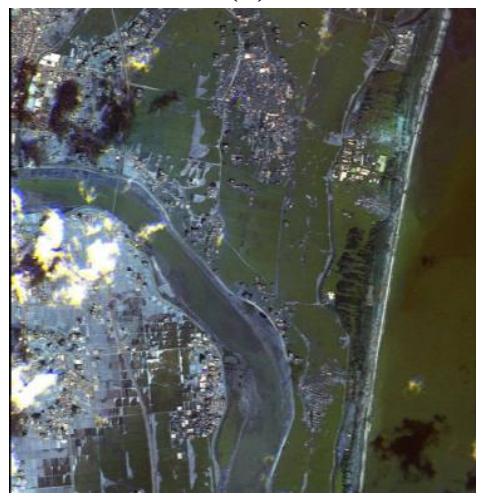

Figure10. RapidEyes satellite data (a) pre tsunami and (b) post tsunami.

The first image acquisition is pre-tsunami event while second image acquisition is posted the tsunami event of 2011. Evidently, huge damages have been caused by the Japan tsunami 2011 (Figure 10b). This is contributed due to fact that Rapid Eye has five distinct bands of the electromagnetic spectrum: Blue $(440-510 \mathrm{~nm})$, Green $(520-590 \mathrm{~nm})$, Red $(630-690 \mathrm{~nm})$, Red-Edge $(690-730 \mathrm{~nm})$ and NearInfrared (760-880 nm). The nominal resolution on the ground is 5 meters, corresponding to NIIRS 2 with daily revisit time [15].

The HI implemented to visible bands in fact, the infrastructures and urban are accurately imaged in visible bands compared to NIR. The comparison between 2-D phase unwrapping; 3-D phase unwrapping; and 4-D phase unwrapping is shown in Figure 11. The hologram interferometry fringe pattern is more vibrant by using 4-D phase unwrapping as compared to other phase unwrapping dimensions i.e. 2-D and 3-D. Particularly, the complete cycle of hologram interferometry fringe pattern is certain with the 4-D phase unwrapping algorithm. In this regard, 4-D holographic interferometry fringes produced by using Hybrid Genetic Algorithm based on Pareto Optimal Solutions. It is interesting to find that the proposed algorithm has produced clear feature detection of infrastructures. In fact, the proposed algorithm has minimized the error in interferogram cycle due to the low coherence in water and vegetation zones and along the coastline due to tsunami impact. This could be the improvement of such previous work of Hussein et al. [5], Karout [7]; Marghany $[8,9,10,11,12,13]$.

(a)

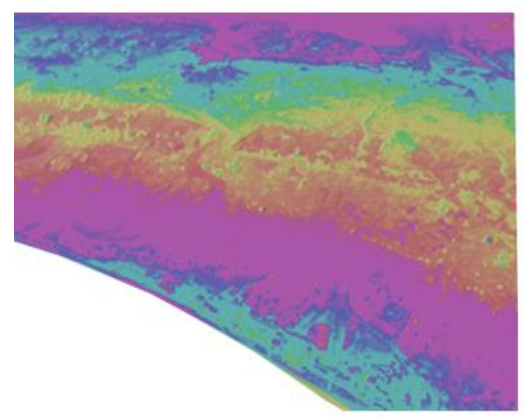

(b)

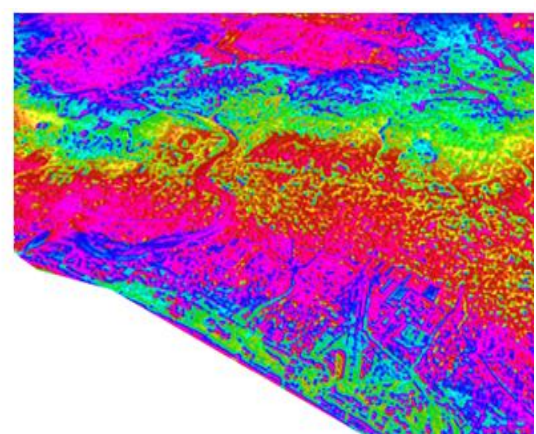

(c)

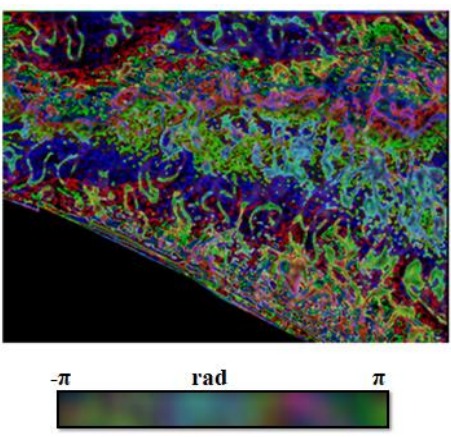

Figure11. Phase unwrapping (a)2-D;(b)3-D; and (c) 4-D. 
Figure 11c represented 4-D phase unwrapping, which typically different from both 2-D and 3-D phase unwrapping. The 4-D phase unwrapping adds more information of water tsunami run-up as indicated in the drainage pattern. This velocity of water motion can be detected in 2-D and 3-D phase unwrapping. Figure 12 shows the 4-D hologram interferometry. It shows that the tsunami run-up was approximately $9 \mathrm{~m}$ height and caused deformation of $20 \mathrm{~m}$ along coastline and inland. It is shown also $7 \mathrm{~m}$ of water flow through the river.

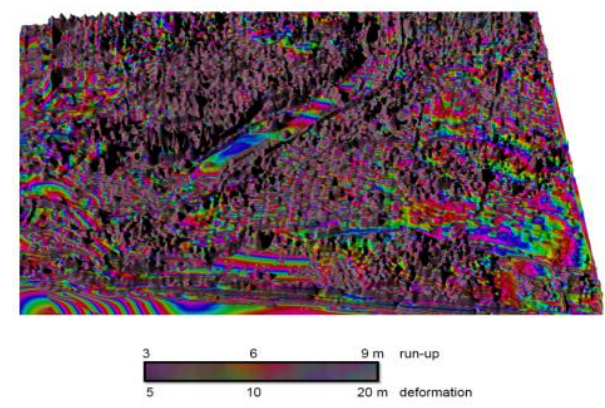

Figure 12. 4-D Hologram Interferometry.

Figure 13 shows the 4-D visualization derived from hologram interferometry. Obviously, the 4-D visualization distinguishes between infrastructures and buildings. With this regard, coding 2-D of RapidEye data into 4-D can visualize much information about urban features, i.e., building, infrastructures, and roads in spite of the great damages caused by the tsunami wave. In addition, it is clear that 4-D shows details of turbulent water flow through the river. Indeed, the hologram interferometry is considered as a deterministic algorithm which is described here to optimize a triangulation locally between two different points. This corresponds to the feature of deterministic strategies of finding only sub-optimal solutions usually. This confirms that optical interferometry is a robust means for evaluating displacements of the order of a wavelength of electromagnetic spectra. Therefore, involving a hologram can assist in recovering the matter of specular reflection beside permitting tiny changes of random, diffusely replication, and objects to be identified [22].

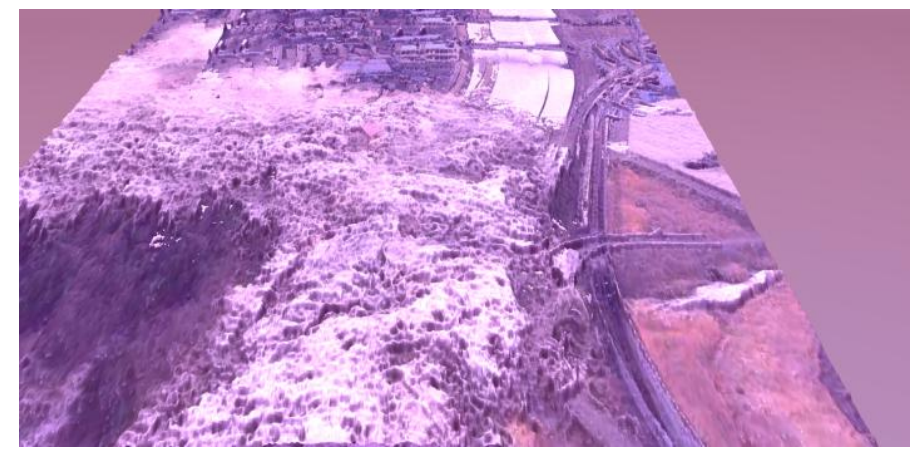

Figure 13. 4-D simulated from Hologram Interferometry

The visualization of the infrastructures is sharp by 4-D phase unwrapping based Hybrid Genetic Algorithm. These results deliver an excellent promising for 4-D reconstruction using 4-D phase unwrapping of hologram interferometry. This study is improving the work done by Marghany [14]. Furthermore, adding fourth coordinate $p$ in the mathematical formula of 3-D HI shaped an excellent 4-D object visualization.

HGA assists to deliver 4-D image and not only discriminating the individual relaxed deformation but also sorting out impeding objects in 4-D. Generally, HGA corresponds the phase of the wrapped phase with guessed unwrapped phase to verify the best representation of the unwrapped phase. Consistent with Karout [7], Saravana et al., [18] a genetic algorithm is used to approximate the coefficient of an $\mathrm{n}^{\text {th }}$ order polynomial which is considered as the best estimates for the unwrapped phase map. With this regard, it minimizes the variance between the unwrapped phase gradient and the wrapped phase gradient. The genetic algorithm uses an initial solution to speed convergence. The initial solution is achieved by unwrapping using a simple unwrapping algorithm and estimating the parameters of the polynomial using weighted least squares multiple regression. Furthermore, 4-D results obtained with the hybrid genetic algorithm exceed the performance of the 4-D phase unwrapping of hologram interferometry. In fact, hybrid genetic algorithm hierarchical by the number 
of miss-unwrapped, which is persuaded meander as the best population members created equivalent quantities of misalliances.

\section{CONCLUSION}

The 4-D phase unwrapping methods outlined are applicable to reconstruct 4-D visualization of tsunami effects using RapidEye data. The involving of Hybrid genetic algorithm to optimize the modification formula of hologram interferometry can permit the RapidEye data to be coded into 4-D. The results show that the varieties of 2-D objects in RapidEye data can be visualized in 4-D. The fine structures of roads, urban, buildings and infrastructures are well visualized in 4-D. In conclusion, the modification and optimization of hologram interferometry formula hold excellent promise for 4-D object visualization in such optical satellite data of RapidEye.

\section{REFERENCES}

[1] Alday, L., Gaiotto, D., \& Tachikawa, Y., 2010, "Liouville correlation functions from four-dimensional gauge theories", Letters in Mathematical Physics, 91 (2), pp. 167-197.

[2] Anne, D., W., 2013, "Moral Authority, Men of Science, and the Victorian Novel". Cambridge University Press

[3] De la Torre-Ibarra, M. H., Santoyo, F. M., \& Moreno, I., 2010, "Digital holographic interferometer using Simultaneously three lasers and a single monochrome sensor for 3D displacement measurements", Optics express,18(19), pp. 19867-19875.

[4] Kohli D, Richard S, Norman K, \& Stein, A, 2012, "An ontology of slums for image-based classification. Computers”, Environment and Urban Systems, 36 (2), pp. 154-163.

[5] Hussein, S. A., Gdeist M, Burton D, \& Lalor M., 2005, "Fast three-dimensional phase unwrapping algorithm based on sorting by reliability following a non-continuous path", Proc. SPIE, 5856,pp. 40.

[6] Haupt, R. L., \& Haupt, S. E., 2004, "Practical genetic algorithms", John-Wiley \& Sons.

[7] Karout, S., 2007, "Two-Dimensional Phase Unwrapping", Ph.D. Theses, Liverpool John Moores University.

[8] Marghany, M., 2012, "Simulation of 3-D Coastal Spit Geomorphology Using Differential Synthetic Aperture Interferometry (DInSAR)"., In I. Padron.,(ed.) Recent Interferometry Applications in Topography and Astronomy, Croatia: InTech - Open Access Publisher, pp. 83-94.

[9] Marghany, M., 2013, "DInSAR technique for three-dimensional coastal spit simulation from radarsat-1 fine mode data", Acta Geophysica, 61,2, pp.478-493.

[10] Marghany, M., 2014, "Simulation of three-dimensional of coastal erosion using differential interferometric synthetic aperture radar", Global NEST Journal, 16, 1, pp 80-86.

[11] Marghany, M. 2014, "Hybrid Genetic Algorithm of Interferometric Synthetic Aperture Radar For ThreeDimensional Coastal Deformation", Frontiers in artificial intelligence and applications: new trends in software methodologies, tools, and technique, 265, pp.116-31.

[12] Marghany, M. 2011, "Modelling shoreline rate of changes using holographic interferometry", Int. J. of Phys. Sci, 6, pp. 7694-7698.

[13] Marghany, M.,2003, "Polarised AIRSAR along track interferometry for shoreline change modeling", In Geoscience and Remote Sensing Symposium, 2003. IGARSS'03. Proceedings. 2003 IEEE International, Vol. 2, pp. 945-947.

[14] Marghany M.,2014,"Hologram interferometric SAR and optical data for fourth-dimensional urban slum reconstruction", CD of 35th Asian Conference on Remote Sensing (ACRS 2014), Nay Pyi Taw, Myanmar 27- 31, October 2014, http://www. a-a-r-s.org/acrs/administrator/components/com.../OS-303\%20.pdf. [Acc ess on August 2, 2016].

[15] Marghany M., 2015, Fourth-dimensional optical hologram interferometry of RapidEye for Japan 's tsunami effects" CD of 36th Asian Conference on Remote Sensing (ACRS 2015), Manila, Philippines, 24 28 October 2015,http://www.a-a-r-s.org/acrs/index.php/acrs/acrs-overview/proceedings-1?view=publicati on \& task=show\&id=1691, Access on August 2, 2016.

[16] Pepe, A., 2012, "Advanced Multitemporal Phase Unwrapping Techniques for DInSAR Analyses", In Padron I. (ed.) "Recent interferometry applications in topography and astronomy". InTech - Open Access Publisher, University Campus STeP Ri, Croatia, pp.57-82.

[17] Smith, J., 2010, "Optimizing remote sensing for disaster monitoring, retrieved May 13, 2005, from http://www.remotesening.net/index.htm.

[18] Saravana, S.S., Ponnanbalam, S.G., \& Rajendran, C. A. 2003, "Multiobjective genetic algorithm for scheduling a flexible manufacturing system", International Journal of Advanced Manufacturing Technology, 22, pp. 229-236. 
[19] Ray, I., 1992, Introducing Einstein's Relativity, Clarendon Press, chp. 22.8 Geometry of 3-spaces of constant curvature, p.319ff, ISBN 0-19-859653-7.

[20] Schwarz O. 2004. Hybrid phase unwrapping in laser speckle interferometry with overlapping windows, Shaker Verlag.

[21] Takeda, M., Ina, H., \& Kobayashi, S.,1982, "Fourier-transform method of fringe-pattern analysis for computer based topography and interferometry", JosA, 72(1), pp.156-160.

[22] Saxby G., 1987: "Practical Holography", Prentice Hall,NJ.

[23] Dawson A, Stewart I, Tsunami Geoscience Progress in Physical Geography. 2007, 31(6)575-590.

[24] Taru T, Japan Experts Warn of Future Risk of Giant Tsunami.http://www.cosmostv.org/2012/04/japanexperts-warn-of-future-risk-of.html. (Accessed 11 September 2016).

[25] Valdes R, Halabrin N, Lamb R How Tsunamis Work.http://science.howstuffworks.com/nature/naturaldisasters/tsunami.htm. (Accessed 11 September 2016).

[26] Marghany M. 2014.Simulation of Tsunami Impact on Sea Surface Salinity along

[27] Banda Aceh Coastal Waters, Indonesia. Advanced Geoscience Remote Sensing. In M. Marghany (ed.), INTECH publisher, Croatia, pp.229-251.

[28] Daily Mail Reporter 2011. Cars, whole houses and even severed feet in shoes: The

[29] vast field of debris from Japan earthquake and tsunami that's floating towards U.S. West Coast. http://www.dailymail.co.uk/news/article-1374520/Japan-earthquake-tsunami-debris-floating-US-WestCoast.html.

[30] Kitanidis,E.,2012.The 2011 Japan Earthquake, Tsunami and Nuclear Disasterhttp://large.stanford.edu/ courses/ 2011/ph240/ kitanidis2/. [Acess $3^{\text {rd }}$ October 2016].

[31] Wikipedia 2016. 2011 Tōhoku, Earthquake and Tsunami. https://en.wikipedia.org/wiki/2011_T\%C5\%8 Dhoku_earthquake_and_tsunami\#cite_note-78. Access $3^{\text {rd }}$ October 2016].

[32] Jones R and Wykes C, 1989. Holographic and Speckle Interferometry.Cambridge University Press.

[33] Joud F., F Verpillat; F Laloë; M Atlan; J Hare; M Gross (2009). "Fringe-free holographic measurements of large-amplitude vibrations".Optics Letters. 34: 3698-3700.

[34] Marghany M. Four-Dimensional Earthquake Deformation Using Ant Colony Based Pareto Algorithm. Communications in Applied Sciences. 2019 Feb 20;7(1).

[35] Marghany M. Advanced Remote Sensing Technology for Tsunami Modelling and Forecasting. CRC Press; 2018 Jul 4.

Citation: Maged Marghany,Four-Dimensional of Japan Earthquake-Tsunami Disaster from Rapid Eye Satellite Data", International Journal of Research in Environmental Science (IJRES), vol. 5, no. 1, pp. 24-34, 2019. Available: DOI: http://dx.doi.org/10.20431/2454-9444.0501005

Copyright: (C) 2019 Authors. This is an open-access article distributed under the terms of the Creative Commons Attribution License, which permits unrestricted use, distribution, and reproduction in any medium, provided the original author and source are credited. 